\title{
De novo prediction of RNA-protein interactions with Graph Neural Networks
}

\author{
Viplove Arora and Guido Sanguinetti
}

September 28, 2021

\begin{abstract}
RNA-binding proteins (RBPs) are key co- and post-transcriptional regulators of gene expression, playing a crucial role in many biological processes. Experimental methods like CLIP-seq have enabled the identification of transcriptome-wide RNA-protein interactions for select proteins, however the time and resource intensive nature of these technologies call for the development of computational methods to complement their predictions. RNA-binding proteins (RBPs) are key co- and post-transcriptional regulators of gene expression, playing a crucial role in many biological processes. Experimental methods like CLIP-seq have enabled the identification of transcriptomewide RNA-protein interactions for select proteins, however the time and resource intensive nature of these technologies call for the development of computational methods to complement their predictions.
\end{abstract}

\section{Introduction}

RNA-protein interactions are fundamental in the regulation of gene expression. RNA-binding proteins (RBPs) are key in RNA splicing, processing, export, localization, and regulation of translation. Despite their importance, RNA-protein interactions are still relatively understudied, when compared with the DNA-protein interactions which are involved in the initiation and regulation of transcription. Many proteins with previously unsuspected RNA-binding properties are still being discovered, and more than 2000 human proteins have been experimentally determined to bind RNA (Brannan et al., 2016; Hentze et al., 2018; Liu et al., 2019). However, due to the difficulty of experimentally determining interactions between individual proteins and individual transcripts, the number of RBPs for which the identity of their interaction partners is known is much lower.

A major breakthrough in the study of RNA-protein interactions was the development of highthroughput techniques such as CLIP-seq (cross-linking and immunoprecipitation followed by next generation sequencing) (Licatalosi et al., 2008). CLIP-seq enables the isolation of RBPs and the fragments of RNA which are bound to them, much in the way that ChIP-seq is used to determine regions of DNA bound by transcription factor proteins. Thanks to improvements in the technology such as the development of the eCLIP protocol (Van Nostrand et al., 2016), huge numbers of RBP binding sites are being verified. Despite these advances, practical and conceptual hurdles mean that we are still very far from a comprehensive mapping of the network of RNA-protein interactions. First of all, such networks are intrinsically condition dependent (for example, simply because specific transcripts might be present or absent in different conditions). Secondly, the experimentally determined interactions are inevitably noisy, meaning that both false positives and false negative results are likely. Thus, there is a need for computational methods to complement experimental techniques by filtering noise and predicting interactions for new conditions as well as new RBPs. Here, we consider the problem of predicting RNA-protein interaction (RPI) pairs adopting a machine learning perspective, where a model is trained on currently validated interactions, using RNA and protein sequences as inputs.

Most current predictive methods focus on the narrower problem of predicting binding sites for a specific protein within RNAs, often combining sequence and secondary structure of the target transcript (Maticzka et al., 2014; Kazan et al., 2010; Alipanahi et al., 2015; Uhl et al., 2021). Due to the lack of large scale CLIP-seq datasets in the past, most methods for predicting RNA-protein interaction pairs 
have only been trained on small datasets (Pan et al., 2019, Section 4.1). RPIseq (Muppirala et al., 2011) uses the sequence information of RNAs and proteins to predict interactions using SVM and random forests as classifiers. catRAPID omics (Agostini et al., 2013) uses the physiochemical properties of sequences to predict RNA-protein interactions on a genome-wide scale. Deep learning-based methods were also proposed (e.g. IPMiner (Pan et al., 2016), RPI-SAN (Yi et al., 2018), RPIFSE (Wang et al., 2019), RNAcommender (Corrado et al., 2016), and ELM* (Wang et al., 2018)) but due to data paucity they were not trained in an end-to-end way and usually relied on advanced feature engineering.

In this paper, we propose to exploit new, large-scale eCLIP datasets (Van Nostrand et al., 2020) to shift the problem of RNA-protein interaction prediction to the network level, i.e. attempting to predict the whole network of RNA-RBP interactions in an organism in a particular condition in an end-to-end way. We use graph neural networks for predicting RNA-protein interactions, moving away from the paradigm of predicting the targets of a single protein towards leveraging whole network information. Our model is trained using high-throughput CLIP-seq data generated as part of the ENCODE project (Van Nostrand et al., 2020) to predict missing links in the RPI network. We also show that the model can be used to predict the interactions for previously unseen proteins as well as transfer the knowledge to a network observed under different biological conditions. We achieve this by using the similarity between the sequence-based features of proteins to elicit the embedding for a previously unseen protein.

\section{Materials and methods}

\subsection{Dataset}

CLIP-seq experiments can provide genome-wide binding sites for RBPs. To retrieve these binding sites, the CLIP-seq reads are first mapped to a reference genome, followed by identification of the peaks of reads using peak calling softwares. These peaks correspond to RBP binding sites based on a certain predefined cutoff, which can be used to identify the set of RNAs a protein binds to.

To construct the benchmark datasets, we use the eCLIP dataset for two cell lines (HepG2 and K562) generated as part of the ENCODE project phase III (Van Nostrand et al., 2020). We use the highly reproducible peaks identified from the two replicates of the eCLIP data using the Irreproducibility Discovery Rate (IDR) framework (Li et al., 2011) to obtain the binding regions. The gene corresponding to the binding site is obtained by using the bedtools intersect function (with a pre-defined minimum overlap between genomic features, we use $50 \%$ in this study) with the human genome (Gencode v19 is used). Repeating the procedure for each protein in the eCLIP dataset we obtain a bipartite network $G=(V, E, \mathbf{X})$ of RNA-protein interactions for a particular cell line. The node set $V=R \cup P$, where $R$ is the set of RNAs and $P$ is the set of RBPs, and $\mathbf{X}$ is a matrix of node features (see Section 2.1 .2 for further details).

The final graph for the K562 cell line consists of 14665 nodes (120 proteins and 14545 RNAs) with 144527 interactions between proteins and RNAs. The mean (out) degree of proteins is 1204.39 with a standard deviation of 1304.64, while the mean and standard deviation of the RNA (in) degree are 9.94 and 10.27, respectively. For the HepG2 cell line, the graph contains 15018 nodes (103 proteins and 14915 RNAs) and 145509 edges. The mean (out) degree of proteins is 1412.71 with a standard deviation of 1380.69 , while the mean and standard deviation of the RNA (in) degree are 9.76 and 10.03, respectively. Figure S1 plots the distribution of protein and RNA degrees for the RPI networks in the two cell lines.

\subsubsection{Negative Interactions}

CLIP-seq experiments provide information about binding sites from the peaks of reads, but they do not provide any information about unbound sites. False negatives in a well-known problem in CLIPseq data because of absence or low concentration of transcripts in the cell line used for experiments (Uhl et al., 2017; Maticzka et al., 2014). Appropriately defining negative samples is important for training machine learning algorithms (Mikolov et al., 2013a). Negative interactions play a crucial role 
in computation of the loss function, and recent work by Ying et al. (2018) has shown that appropriately choosing the negative samples can boost the performance of a link predictor. This becomes even more important for bipartite networks where random sampling two unconnected nodes can produce edgetypes that do not exist in the data (RNA-RNA for example).

To tackle this issue, the following two strategies have been commonly used to construct negative samples from CLIP-seq data: (i) use the regions where no binding sites are located as negative samples, or (ii) use randomly shuffled nucleotides in the positive sequences as negative samples. We augment the first strategy by utilizing the RNA-seq transcript abundance data to identify RNAs that have transcripts per million (TPM) counts more than the median value in the cell line but still do not have any peaks with the corresponding protein to define negative samples. This strategy allows the model to learn from highly likely unbound sites of real RNA sequences and alleviates the problem of false negatives described above. Using RNA-seq we identify reliable non-interacting RNAs for each protein and consequently use these negative samples to define the training and test sets.

\subsubsection{Node Features}

Node features are essential for training GNNs as they allow the neighborhood aggregation process to capture the hierarchical non-linearities in the network data. The sequences of proteins and RNAs can be encoded as numeric vectors for training machine learning models. To extract features from RNAs, the k-mer representation is widely used for predicting RNA-protein interaction and they mainly have two forms of representation: (i) one-hot vector, which is a bit vector that consists of all zeros except for a single dimension, and (ii) k-mer frequency vector, which is a vector consisting of frequencies of all k-mers, similar to bag-of-words (BOW) in natural language processing. We use the following feature extraction methods, which have been successfully used by previous methods for predicting RNA-protein interactions (Muppirala et al., 2011; Pan et al., 2016):

- Proteins: Conjoint triad descriptors (Shen et al., 2007) abstract the features of proteins based on the classification of amino acids according to their dipoles and volumes of the side chains. Each protein sequence is encoded using a normalized 3-gram frequency distribution extracted from a 7-letter reduced alphabet representation.

- RNA: k-mer frequency distribution counts the frequency of individual k-mers (AAA, AAC, ..., UUU are 3-mers) in a given RNA sequence. It is the simplest feature extraction method for RNAs, where $k$ can be used as a hyperparameter. We use $k=6$ for our experiments.

Following the above feature extraction method, we obtain $7^{3}=343$ and $4^{6}=4096$ dimensional feature vectors for proteins and RNAs respectively. For aggregating the features in a GNN, node features should have the same dimensionality. To achieve this, we use the first 100 principal components of protein and RNA features to define the node features $\mathbf{X}$ for the network $G$.

Features can also be extracted by treating RNA and protein sequences as a special kind of language, where k-mers can be treated as words and sequences as sentences. Natural language processing techniques such as word2vec (Mikolov et al., 2013b) can then be used to learn embeddings for protein and RNA sequences (Asgari and Mofrad, 2015). Results for this alternative feature extraction scenario can be found in the Supplementary information.

\section{$2.2 \quad$ Graph Neural Networks}

In recent years, GNNs have become an indispensable tool for applying neural networks to the graph domain (see Wu et al. (2020) and Zhou et al. (2020) for recent reviews). The GNN architecture creates a non-linear permutation invariant transformation function on node, edge and graph features, which can be optimized for performing downstream learning tasks. The neighborhood aggregation process of GNNs allows us to capture the hierarchical non-linearities in network data, thus learning low dimensional embeddings for the nodes of a graph. The ability to learn from the entire network of RNA-protein interactions enables us to build a single end-to-end model for predicting RNA-protein 


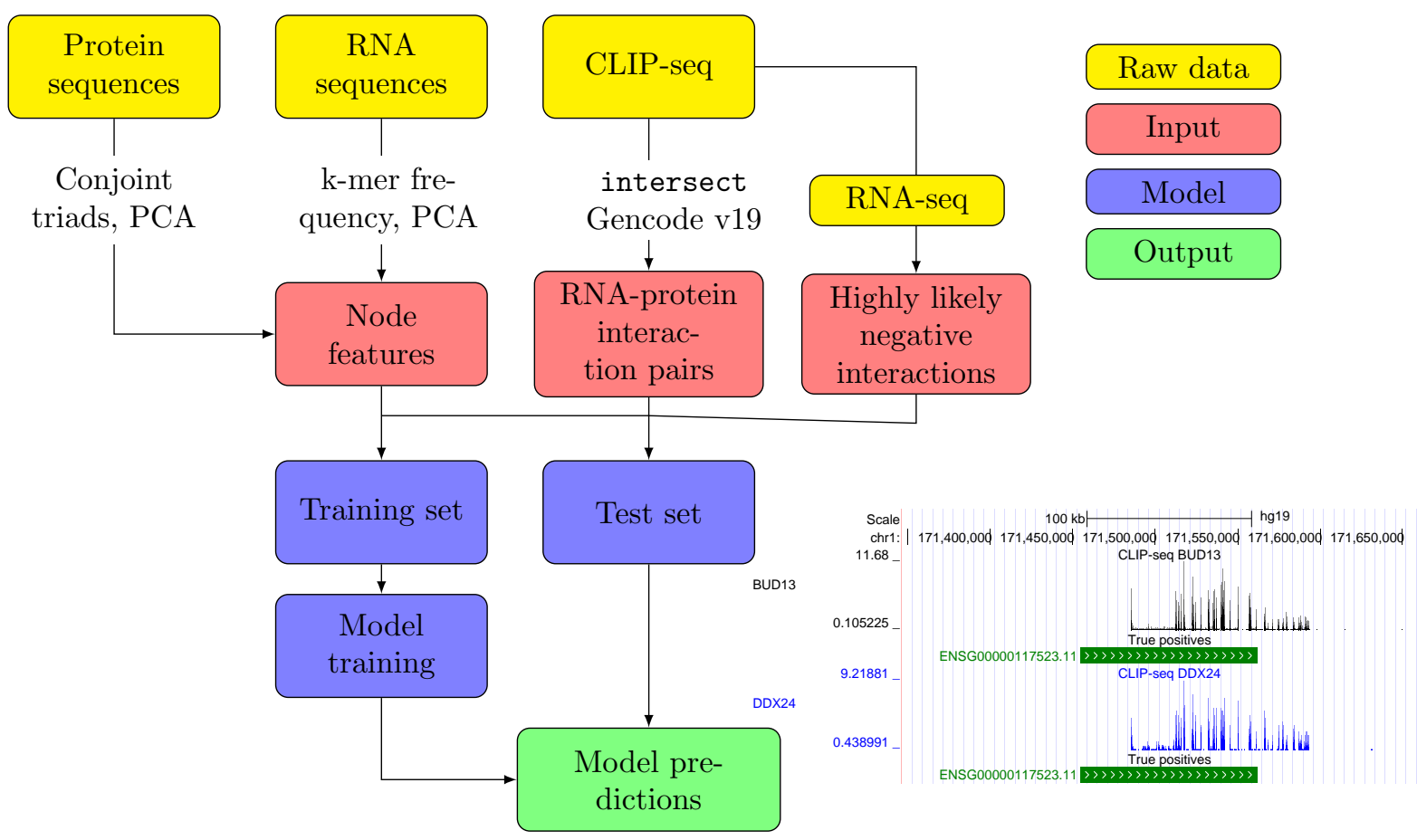

Figure 1: Pictorial representation of the framework presented in this paper: The raw data is transformed to obtain node features, positive and negative interactions, which serve as input for the GNN. The trained model is used for making predictions as shown using the genome tracks.

interactions. Further, the GNN architecture facilitates the aggregation of information from distant neighbors such as other proteins, thus learning better node representations.

A lot of the existing GNN architectures can be directly translated into the framework of message passing neural networks (Gilmer et al., 2017), where each node sends and receives messages (using function $\left.M_{k}(\cdot)\right)$ from its neighbors, and subsequently updates (using function $U_{k}(\cdot)$ ) its own node state:

$$
\mathbf{h}_{v}^{(k+1)}=U_{k}\left(\mathbf{h}_{v}^{(k)}, \sum_{u \in N(v)} M_{k}\left(\mathbf{h}_{v}^{(k)}, \mathbf{h}_{u}^{(k)}, \ldots\right)\right),
$$

where $\mathbf{h}_{v}^{(k)}$ is the hidden representation of node $v$ in layer $k, M_{k}(\cdot)$ and $U_{k}(\cdot)$ are functions with learnable parameters, $N(v)$ is the set of neighbors of node $v$, and ... represent other features (such as edge features) that can be added to the message passing process. Node features, if available, can be used as the initial hidden representation $\mathbf{h}_{v}^{(0)}=\mathbf{x}_{v}$ for a node. After $K$ message passing layers, node embedding $\mathbf{z}_{v}$ is produced for each node $v$ as the final output, which can then be used for node, link, or graph level prediction tasks. The functions $M_{k}(\cdot)$ and $U_{k}(\cdot)$ share parameters across nodes, but each node is associated with an individual computation graph defined by its neighbors (Ying et al., 2019). In this paper, we use Graph Convolutional Network (GCN) (Kipf and Welling, 2017) with $K=2$ as the GNN model (results for other GNNs can be seen in the Supplementary information). GCN bridges the gap between spectral and spatial approaches for performing convolution over graph-based data (Wu et al., 2020). The graph convolution operation of GCN can be written as:

$$
\mathbf{H}^{(k+1)}=\sigma\left(\hat{\mathbf{D}}^{-1 / 2} \hat{\mathbf{A}} \hat{\mathbf{D}}^{-1 / 2} \mathbf{H}^{(k)} \Theta^{(k)}\right),
$$

where $\hat{\mathbf{A}}=\mathbf{A}+\mathbf{I}$ is the adjacency matrix with self loops and $\hat{\mathbf{D}}$ is the diagonal degree matrix corresponding to $\hat{\mathbf{A}}$. $\mathbf{H}^{(k)}$ is the matrix containing the hidden representation of nodes at layer $k$ with $\mathbf{H}^{(0)}=\mathbf{X}, \boldsymbol{\Theta}^{(k)}$ are the model parameters at layer $k$, and $\sigma(\cdot)$ is an element-wise activation function. Comparing Equations 1 and 2, for GCN the message and update functions take the following form (Gilmer et al., 2017): 


$$
\begin{gathered}
M_{k}\left(\mathbf{h}_{v}^{(k)}, \mathbf{h}_{u}^{(k)}\right)=(\operatorname{deg}(v) \operatorname{deg}(u))^{-1 / 2} A_{v u} \mathbf{h}_{v}^{(k)} \\
U_{k}\left(\mathbf{h}_{v}^{(k)}, \mathbf{m}_{v}^{(k+1)}\right)=\sigma\left(\boldsymbol{\Theta}^{(k)} m_{v}^{(k+1)}\right) \\
\mathbf{m}_{v}^{(k+1)}=\sum_{u \in N(v)} M_{k}\left(\mathbf{h}_{v}^{(k)}, \mathbf{h}_{u}^{(k)}\right) .
\end{gathered}
$$

To add some biological context to the model, we consider the following different settings for GCN:

- RNA-seq experiments provide high resolution information about the presence and quantity of all the RNAs in a given biological sample. RNA-seq can tell us which genes are turned on in a cell, and what their level of transcription is (Ozsolak and Milos, 2011). Thus, one would assume that RNA abundance would be a good indicator of an RNA's ability of being bound by a protein. In the $S e q$ variant of GCN, we append $\log (1+\mathrm{TPM})$ to the final node embedding $\mathbf{Z}$ of RNAs (set to -1 for all proteins) obtained after GNN layers. The modified embeddings are then used for computing the loss and predicting interactions.

- As described in Section 2.1.1, negative sampling plays an important role in the training of machine learning models. In the default training regime for GCN, negative edges are sampled uniformly at random from the set of possible negative interactions, which could result in a case where more negative interactions are sampled for a protein with a low degree. To deal with this issue, we consider structured negative (SN) sampling, where negative interactions for a protein are sampled proportional to its degree.

Figure 1illustrates the general workflow of our proposed framework.

\subsection{Link prediction and evaluation metrics}

The current knowledge of interactions in biological networks is often incomplete, which makes predicting missing interactions an important task (Muzio et al., 2020). Link prediction is often framed as a semi-supervised learning problem, where the known links in a network are used to predict additional interactions. Given the importance of RNA-protein interactions and the challenges associated with experimental methods, predicting potential interactions using computational models can compliment our current knowledge (Corrado et al., 2016). While most existing studies focus on transductive link prediction (both nodes are in the graph), inductive (or out-of-sample) link prediction can prove immensely valuable for new proteins.

In a GNN for link prediction, the message passing procedure described in Section 2.2 is used to compute individual node representations $\mathbf{z}_{u}$, following which a function $p_{u v}=f\left(\mathbf{z}_{u}, \mathbf{z}_{v}\right)$ can be used to predict the probability of the link $(u, v)$. The model can be trained to maximize the likelihood of reconstructing the true adjacency matrix $\mathbf{A}$ using the binary cross entropy loss:

$$
\mathcal{L}(u, v)=-A_{u v} \log \left(p_{u v}\right)-\left(1-A_{u v}\right) \log \left(1-p_{u v}\right) .
$$

Splitting networks into training and test subnetworks is not trivial in link prediction problems. While performing the train-test split of edges, we need to make sure that every node in the training network has a non-empty set of neighbors so that the GNN can learn appropriate representations using the message passing process shown in Equation 1. To ensure this, test edges are sampled for each RNA while making sure that it stays connected in the training network.

Link prediction is a binary classification task and the performance of an algorithm can be evaluated using different metrics. These metrics can be divided into two broad categories: fixed-threshold metrics and threshold curves (Yang et al., 2015). In research context, we generally do not have a reasonable threshold, which is why threshold curves and scalar measures summarizing them are widely used in the literature (Davis and Goadrich, 2006; Clauset et al., 2008; Lichtenwalter et al., 2010). In this paper, we use area under the receiver operating characteristic (AUROC), and average precision 
(AP) to evaluate performance of different methods on the link prediction task. The receiver operating characteristic (ROC) curve represents the performance trade-off between true positives and false positives at different decision boundary thresholds. AUROC reflects the probability that a randomly chosen positive instance appears above a randomly chosen negative instance. AP summarises the precision-recall curve, and is a better measure for a highly imbalanced dataset (Davis and Goadrich, 2006; Yang et al., 2015). AP can be computed using the following formula:

$$
A P=\sum_{n}\left(\alpha_{n}-\alpha_{n-1}\right) \beta_{n}
$$

where $\beta_{n}$ and $\alpha_{n}$ are the precision and recall at the $n^{\text {th }}$ threshold.

\subsection{Prediction tasks}

The models are evaluated under three different scenarios: (i) we assume that some percentage of the RNA-protein interactions are missing in Section 3.1? (ii) we perform leave-one-protein-out experiments in Section 3.2, assuming the availability of full interaction information for the remaining proteins; and (iii) transfer learning of RNA-protein interactions from a source cell line to a target cell line in Section 3.3. In scenarios (ii) and (iii), we use the similarity between the sequence-based features of proteins to elicit the embedding for a previously unseen protein. Using machine learning terminology, we refer to scenario (i) as transductive learning (as the set on which predictions are needed is part of the graph), scenario (ii) as inductive learning, and scenario (iii) as transfer learning.

\section{Results and discussion}

Our models were trained using the large-scale eCLIP datasets for RNA-protein interactions extracted from the ENCODE project (see Section 2.1). We use RNAcommender (Corrado et al., 2016) as the baseline model to compare against our GNN-based approach. RNAcommender is a recommender system capable of suggesting genome-wide RNA targets for unexplored RBPs using interaction information available from high-throughput experiments performed on other proteins. In our evaluations, we use the features described in Section 2.1.2 instead of the advanced feature engineering (protein domain composition and the RNA predicted secondary structure) used in the original implementation of RNAcommender (Corrado et al., 2016). For GCN, we consider the settings described in Section2.2, (i) GCN in vanilla setting; (ii) Seq, GCN with RNA-seq; (iii) SN, GCN with structured negative sampling; and (iv) Seq.SN, GCN with RNA-seq and structured negative sampling.

\subsection{Transductive link prediction}

For the first evaluation of the models, we consider the scenario when varying percentage of positive edges are removed from the RPI network. An equal number of negative interactions, as defined in Section 2.1.1. for the test set can be sampled either uniformly at random, or proportional to the degree of each protein. The second setting is considerably harder in practice because the network has to implicitly learn the degree information from training data. On the other hand, the harder setting is likely to be more representative of true biological missing data. We use the AUROC on the validation set to select the best model. In this section, we only present results for the 'hard' setting for negative interactions in the test set (results for the 'easy' case can be found in supplementary information Table (55). We run all experiments 10 times and report the average results and standard deviations, highlighting in boldface the best results (determined using two sample t-tests) in tables.

A hyperparameter that needs to be tuned for GCNs is the dimension of the final embedding of the nodes. In Figure 2, we plot the performance of three different variants of GCN as the final embedding dimension is varied (the hidden dimension is kept constant at 50). While all methods for all embedding dimensions provide results which are clearly better than random predictors, the trend shows a clear peak at dimensions between 5 and 10 for all methods; we therefore choose 10 as the final embedding size for all subsequent evaluations of GCN under different settings. 


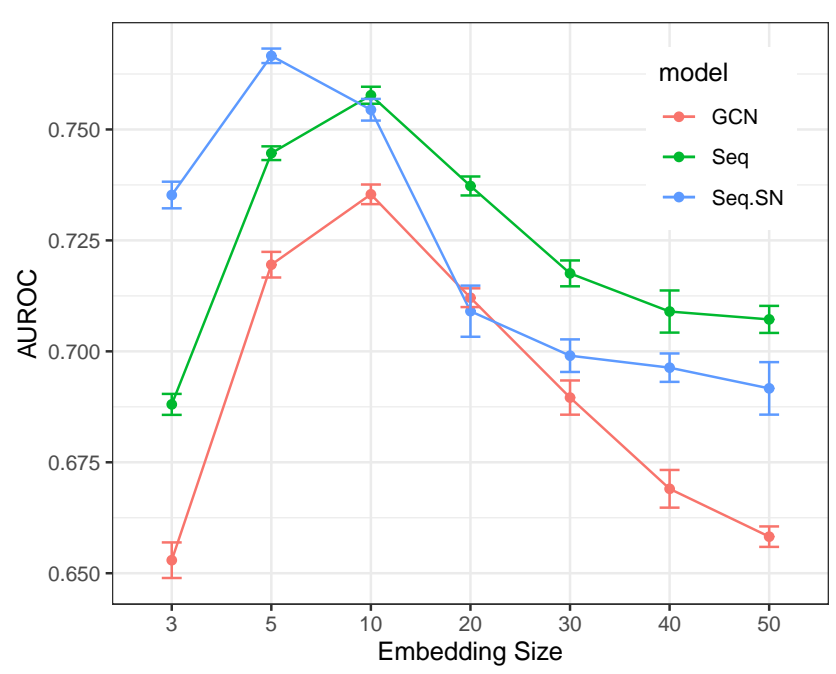

Figure 2: Comparing the performance of various GCN settings while varying the size of the final embedding. The test set contains $20 \%$ edges and the hidden embedding size is set to 50 . The error bars show the standard deviation on 10 independent trials.

Table 1: Comparing the AUROC for transductive learning setting in K562 cell line with varying percent of edges in the test set (validation set contains $10 \%$ edges in all cases). The bold marker denotes the best performing model and \pm denotes the standard deviation on 10 independent trials.

\begin{tabular}{rrrrr}
\hline Test & RNAcommender & GCN & Seq & Seq.SN \\
\hline $10 \%$ & $0.604 \pm 0.003$ & $0.751 \pm 0.002$ & $\mathbf{0 . 7 6 8} \pm 0.004$ & $0.759 \pm 0.003$ \\
$20 \%$ & $0.601 \pm 0.004$ & $0.736 \pm 0.002$ & $\mathbf{0 . 7 5 7} \pm 0.002$ & $0.755 \pm 0.002$ \\
$30 \%$ & $0.595 \pm 0.004$ & $0.717 \pm 0.002$ & $\mathbf{0 . 7 4} \pm 0.002$ & $\mathbf{0 . 7 3 9} \pm 0.002$ \\
$40 \%$ & $0.594 \pm 0.005$ & $0.717 \pm 0.003$ & $\mathbf{0 . 7 4} \pm 0.001$ & $0.739 \pm 0.002$ \\
$50 \%$ & $0.586 \pm 0.005$ & $0.709 \pm 0.003$ & $\mathbf{0 . 7 3} \pm 0.002$ & $\mathbf{0 . 7 3} \pm 0.001$ \\
\hline
\end{tabular}

The results in Table 1 and 2 show the performance of different models on the transductive link prediction task on the RPI networks of K562 and HepG2 cells respectively. The tests are performed varying the percentage of edges in the test set from $10 \%$ to $50 \%$ (full results can be found in the Supplementary information). Our results show that our GCN-based models consistently outperform RNAcommender in the task of transductive link prediction by a clear margin. As expected, the performance drops as we increase the number of edges in the test set (thus decreasing the size of the training set), however for all sizes of training set the performance of the GCN approach (in all its variants) remains above $70 \%$ AUROC. Within the different GCN settings, we see that using RNAseq consistently improves the predictive performance of the model in all cases (this effect is more pronounced when using word2vec based node features, as seen in Table S4).

Results for the simpler setting when negative test edges are selected at random are shown in the Supplementary Information. Here we see a significant improvement for both RNAcommender and the GCN approaches, with the latter achieving test accuracies surpassing 90\% (in some cases substantially so).

\subsection{Inductive link prediction}

The ability to make de novo predictions of RNA-protein interactions is one of the biggest motivations for developing computational models for this problem. This is known as the problem of inductive (or out-of-sample) link prediction in the GNN community. Such analysis is particularly valuable as the model predictions can serve as a proxy for proteins for which high-throughput data is not currently available. In this setting, we create a training network $\bar{G}$ by removing interaction data for the test and validation proteins but assume that the model has access to the raw features of the unseen proteins. The trained model then computes the embedding for the new proteins using normalized 
Table 2: Comparing the AUROC for transductive learning setting in HepG2 cell line with varying percent of edges in the test set (validation set contains 10\% edges in all cases). The bold marker denotes the best performing model and \pm denotes the standard deviation on 10 independent trials.

\begin{tabular}{rrrr}
\hline Test & GCN & Seq & Seq.SN \\
\hline $10 \%$ & $0.771 \pm 0.003$ & $\mathbf{0 . 7 9 8} \pm 0.003$ & $0.779 \pm 0.003$ \\
$20 \%$ & $0.762 \pm 0.003$ & $\mathbf{0 . 7 9} \pm 0.002$ & $0.771 \pm 0.005$ \\
$30 \%$ & $0.748 \pm 0.004$ & $\mathbf{0 . 7 8} \pm 0.002$ & $0.765 \pm 0.003$ \\
$40 \%$ & $0.742 \pm 0.004$ & $\mathbf{0 . 7 7 2} \pm 0.002$ & $0.762 \pm 0.005$ \\
$50 \%$ & $0.734 \pm 0.001$ & $\mathbf{0 . 7 6 3} \pm 0.002$ & $0.758 \pm 0.002$ \\
\hline
\end{tabular}

feature similarity $\operatorname{sim}\left(\mathbf{x}_{v}, \mathbf{x}_{u}\right)$ (based on Euclidean distance or cosine similarity) to previously seen proteins:

$$
\mathbf{z}_{v}=\sum_{u \in \bar{P}} \operatorname{sim}\left(\mathbf{x}_{v}, \mathbf{x}_{u}\right) \mathbf{z}_{u}
$$

where $\bar{P}$ is the set of proteins in the training network $\bar{G}$. We perform experiments with a single protein in the test set (with all highly likely negative interactions as defined in Section 2.1.1), while the protein with the highest feature similarity to the test protein is chosen as the validation protein. This can create a potential class imbalance in the positive and negative interactions in the test and validation sets. We therefore also use the AP metric introduced in Section 2.3, which is a better measure for an imbalanced dataset.
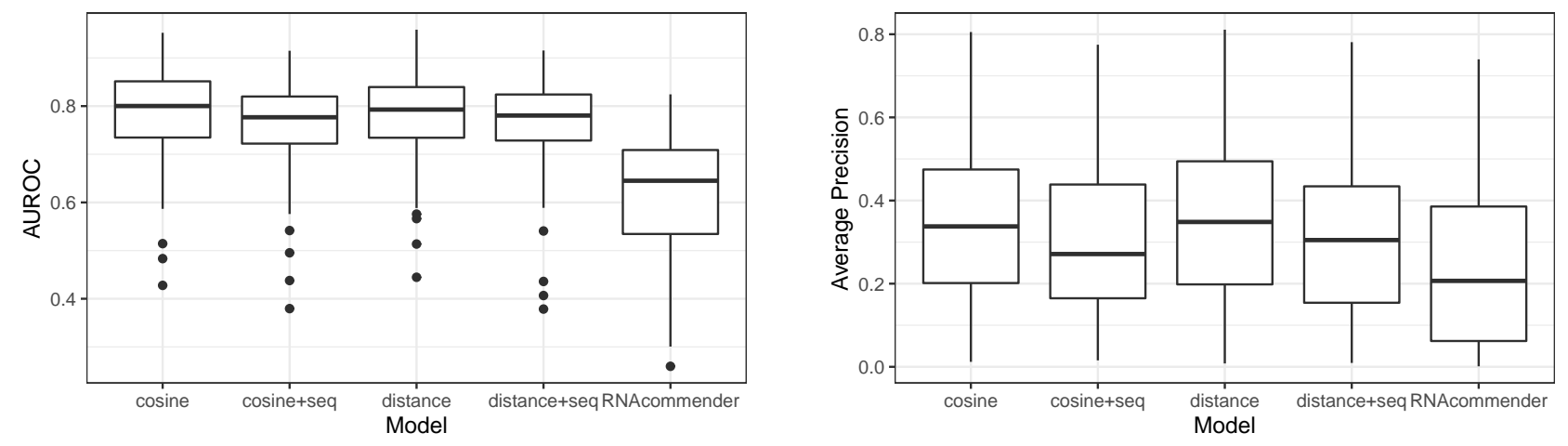

Figure 3: Comparing the performance of various models for de novo prediction in K562 cell line. Each box shows the distribution of mean AUROC (left) or average precision (right) over the entire set of proteins when the model is tested for a single protein in the test set.

Figure 3 compares the performance of different models over the entire set of proteins in the inductive link prediction setting. Each box plots the distribution of mean AUROC/AP for proteins in the K562 cell line (10 replications for each protein). The results show that on average all variations of GCN outperform RNAcommender in the K562 cell line. More specifically, GCN with distancebased similarity outperforms RNAcommender on $93.33 \%$ and $87.5 \%$ of proteins on AUROC and AP, respectively. Among the different settings of GCN, we observe that the choice of similarity function has very little impact on the model performance and even appending RNA-seq to the final embeddings does not seem to substantially improve model performance (although we do see some improvement in the HepG2 cell line, see Figure \$2).

Figure 4 shows genome tracks annotated by the predictions of our model for two proteins, BUD13 and DDX24, in the inductive link prediction task for the K562 cell line. Figures 4a and 4b show example regions corresponding to predicted true positives and true negatives; as expected, true positives correspond to regions with a strong binding signal, while true negatives display a complete absence of signal. Figure 4d and 4e show examples of wrong predictions (false positives and false negatives respectively): both examples show a modest amount of binding, likely representing regions that are borderline cases in the peak calling procedure. This suggests that the incorrect predictions by the 


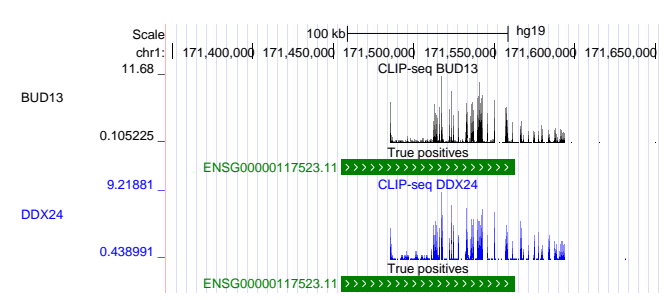

(a) True positives (TP)

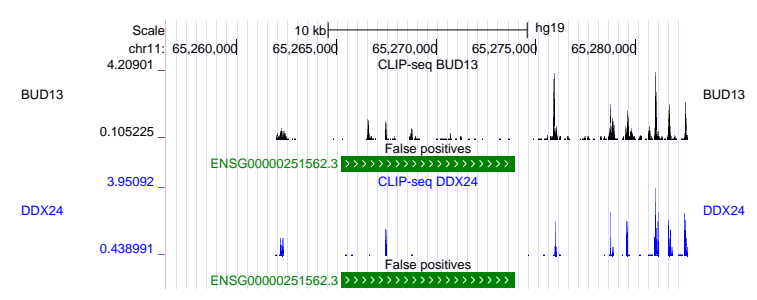

(d) False positives (FP)

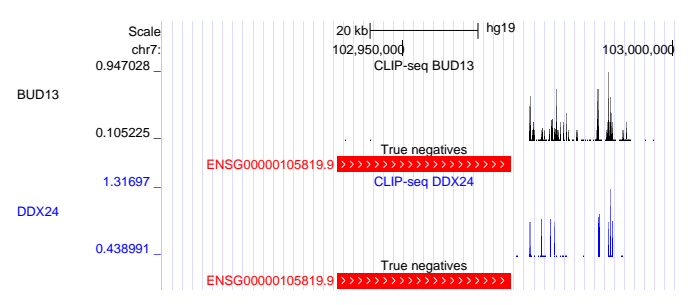

(b) True negatives (TN)

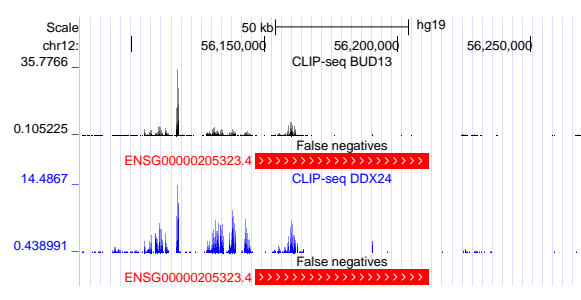

(e) False Negatives (FN)

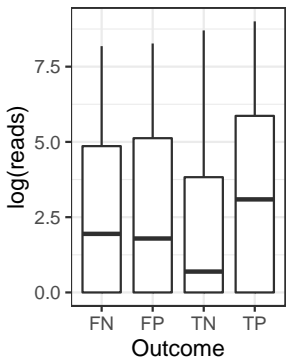

(c) Reads for BUD13

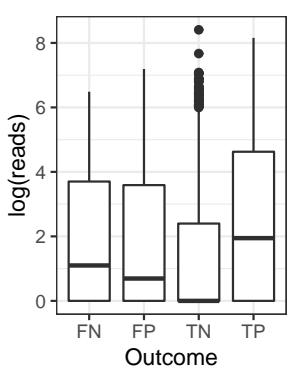

(f) Reads for DDX24

Figure 4: The plots show representative genome tracks produced using the eCLIP data annotated by predictions made by our model under four different outcomes. We consider two proteins, BUD13 and DDX24, in the inductive link prediction task for the K562 cell line. Positive predictions are shown in green and negative predictions in red. We also plot the distribution of reads for the two proteins under the four outcomes.

model may correspond to potentially noisy regions. This point is illustrated globally using Figures $4 \mathrm{c}$ and $4 \mathrm{f}$, which plot the distribution of reads per transcript for the four outcomes. We observe that the true positive and true negative predictions respectively have significantly higher and lower number of reads compared to the other cases, whereas the false positive and false negative predictions by the model have intermediate amounts of reads, potentially corresponding to noisy regions.

\subsection{Transfer learning}

The ENCODE dataset (Van Nostrand et al., 2020) consists of 223 eCLIP experiments for 150 proteins across two different cell lines (K562 and HepG2). This provides an opportunity to perform transfer learning, where a model learnt from the eCLIP data for a source cell line can be used to predict the interactions in a target cell line. This is potentially the most interesting aspect of our approach, as it would permit researchers to obtain a reasonable prediction of an RPI network in new conditions based on minimal information about the target condition.

To train a model for transfer learning, we split the interactions from the source cell line by assigning all interactions from a fixed percentage of randomly chosen proteins to the validation set. Splitting the data in this way allows us to choose a model that has higher predictive power on previously unseen proteins. For creating the test set using the target cell line, we only consider interactions with RNAs that already exist in the source cell line. This allows us to exclusively focus on transfer learning for proteins. Negative interactions in the test set (same number as positive interactions) are sampled uniformly at random from the highly likely negative interactions in the target cell line ('easy' setting in Section 3.1). This is a reasonable assumption because to sample negative interactions proportional to a protein's degree ('hard' setting), we need to have a priori knowledge about its interactions in the target cell line.

To the best of our knowledge, we are the first ones to explore the question of transfer learning for RNA-protein interaction prediction, which is why we only compare the performance of different GCN settings in this section. As in Section 3.2, we use Equation 4 to compute embeddings for the proteins in the test and validation sets. We specifically focus on the use of RNA-seq in transfer learning as it can provide information about RNA abundance in the target cell line. For transfer learning, we append the TPM counts of the source cell line to the RNA embeddings during training, but replace it with the TPM counts in the target cell line for making the final predictions. 
Table 3: Comparing the AUROC for transfer learning from K562 to HepG2 using various GCN settings. We vary the percentage of proteins in the validation set of the source cell line, thus reducing the number of interactions in the training network. The bold marker denotes the best performing model and \pm denotes the standard deviation on 50 independent trials.

\begin{tabular}{lrrrr}
\hline$\%$ val & GCN & SN & Seq & Seq.SN \\
\hline 5 & $0.693 \pm 0.024$ & $0.676 \pm 0.022$ & $\mathbf{0 . 7 2 4} \pm 0.009$ & $0.703 \pm 0.004$ \\
10 & $0.688 \pm 0.026$ & $0.671 \pm 0.013$ & $\mathbf{0 . 7 1 9} \pm 0.006$ & $0.696 \pm 0.006$ \\
20 & $0.677 \pm 0.022$ & $0.66 \pm 0.013$ & $\mathbf{0 . 7 0 4} \pm 0.008$ & $0.685 \pm 0.006$ \\
30 & $0.664 \pm 0.016$ & $0.647 \pm 0.009$ & $\mathbf{0 . 6 8 9} \pm 0.011$ & $0.674 \pm 0.007$ \\
\hline
\end{tabular}

The results in Tables 3 and 4 show that the GCN approach provides a good predictive accuracy even in the transfer learning mode, with AUROC values over $70 \%$ in most of the cases. Additionally, there appears to be a clear advantage of using RNA abundance data in transfer learning as the $S e q$ variant of GCN is the best performing model in most cases. This is intuitively appealing, as it shows that the RNA-seq data clearly conveys some information about the state of the cell which is relevant to the prediction of the RPI network. Nevertheless, it is still very surprising that, even without RNA-seq information, GCNs provide a good predictive performance. To contextualise this observation, in Fig. 5 we compare the ROC curve for the GCN with RNA-seq data to the prediction we would obtain by just assuming the two RPI networks to be the same on the set of proteins/RNAs shared by the two eCLIP experiments (naive transfer). Remarkably, the performance of this naive transfer approach is only marginally better than random, and very considerably worse than the GCN prediction at the same false positive rate. This indicates that the GCN learns primarily robust interactions that are seen in multiple different environments, and which are presumably hard-wired into the protein-RNA sequence features.

Table 4: Comparing the AUROC for transfer learning from HepG2 to K562 using various GCN settings. We vary the percentage of proteins in the validation set of the source cell line, thus reducing the number of interactions in the training network. The bold marker denotes the best performing model and \pm denotes the standard deviation on 50 independent trials.

\begin{tabular}{lrrrr}
\hline$\%$ val & GCN & SN & Seq & Seq.SN \\
\hline 5 & $\mathbf{0 . 7 8 5} \pm 0.037$ & $0.696 \pm 0.022$ & $\mathbf{0 . 7 8 9} \pm 0.017$ & $0.743 \pm 0.031$ \\
10 & $\mathbf{0 . 7 8 5} \pm 0.026$ & $0.696 \pm 0.02$ & $\mathbf{0 . 7 7 8} \pm 0.016$ & $0.749 \pm 0.038$ \\
20 & $\mathbf{0 . 7 7 4} \pm 0.018$ & $0.688 \pm 0.016$ & $0.76 \pm 0.018$ & $0.742 \pm 0.037$ \\
30 & $\mathbf{0 . 7 5 9} \pm 0.021$ & $0.694 \pm 0.018$ & $0.748 \pm 0.016$ & $0.74 \pm 0.032$ \\
\hline
\end{tabular}

We also observe that the performance of GCN degrades as the size of the validation set is increased. This implies that the model learns better by seeing more data from the source cell line instead of just overfitting to the training data.

\section{Conclusion}

Experimental discovery of RPIs has been a major focus of research over the last ten years. After a pioneering period where novel technologies were still being developed, the last few years have seen an effort towards scaling and standardising the technology (Van Nostrand et al., 2016), resulting in the publication in the last year of the first large-scale compendia of RNA-RBP interactions in human cell lines (Van Nostrand et al., 2020).

These technological developments call for a change in the way RPI data are modelled. Earlier approaches (reviewed in e.g. (Pan et al., 2019)) focused on predicting the targets (or the binding sites) of individual RBPs, treating potential target transcripts as i.i.d. observations thus enabling the deployment of machine learning approaches such as CNNs. Now, the availability of binding data from hundreds of RBPs leads to hundreds of correlated prediction tasks, calling for methods that can effectively leverage these structures. In this spirit, our GNN model transfers information between 


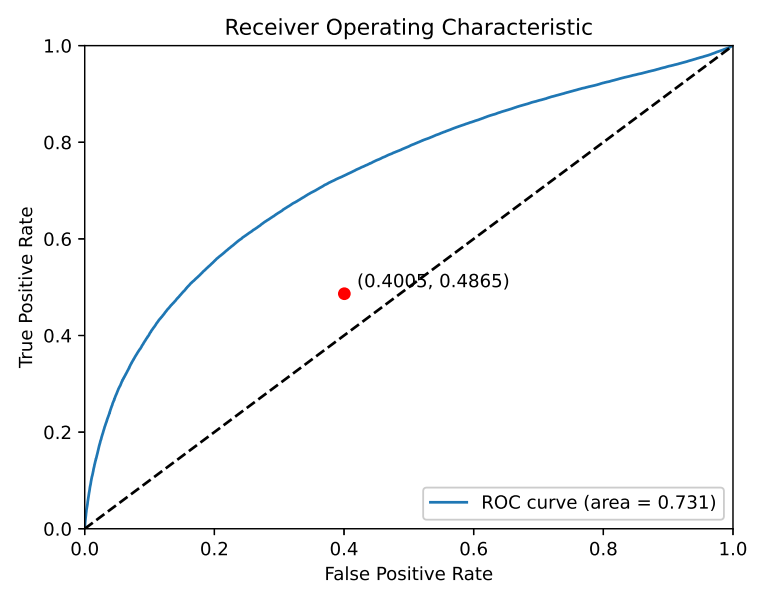

Figure 5: ROC curve for transfer learning from K562 to HepG2 cell line for GCN with RNA-seq. Red dot corresponds to the false positive and true positive rates if the edges from the source cell line are directly transferred to target cell line.

different RBPs binding data, translating the problem of predicting the binding targets of an RBP to predicting the whole RPI network.

Our experiments demonstrate considerable promise in this attempt. Apart from improving by a wide margin on state-of-the-art competitors on the classical tasks of link prediction, our GNN approach also offers strong predictive performance in out-of-sample inductive predictions of the targets of an unseen RBP. Additionally, we show empirically that the GNN approach is also able to perform transfer learning, i.e. predict the RPI network in a different (related) condition starting from RPI data from a well characterised condition, a task that was never attempted to our knowledge.

While we believe GNNs hold much promise for the problem of RPI network prediction, a number of areas for future improvement are clearly open. First of all, proteins (and transcript) are characterised solely by their sequence and their binding partners in our approach, making the task of predicting the full complement of binding partners for a new protein (inductive link prediction) difficult. In principle, the availability of additional node information (for example in the form of protein-protein interactions, or of ontological annotations) could be easily incorporated in the GNN framework, potentially leading to significant improvements. Another area of great interest where improvements are certainly possible is transfer learning. Here the question is to identify suitable covariates which can be used to measure the similarity of different domains. In this paper, we show that the use of RNA-seq data helps in the transfer learning task, presumably because it recapitulates some information on the state of the cell, nevertheless more appropriate task descriptors might be found and integrated in the framework.

\section{Acknowledgements}

The authors want to thank Gianluca Corrado for sharing the implementation details of RNAcommender.

\section{References}

Agostini, F. et al. (2013). cat rapid omics: a web server for large-scale prediction of protein-rna interactions. Bioinformatics, 29(22), 2928-2930.

Alipanahi, B. et al. (2015). Predicting the sequence specificities of dna-and rna-binding proteins by deep learning. Nature biotechnology, 33(8), 831-838.

Asgari, E. and Mofrad, M. R. (2015). Continuous distributed representation of biological sequences for deep proteomics and genomics. PLoS One, 10(11), 1-15. 
Brannan, K. W. et al. (2016). Sonar discovers rna-binding proteins from analysis of large-scale proteinprotein interactomes. Molecular cell, 64(2), 282-293.

Clauset, A. et al. (2008). Hierarchical structure and the prediction of missing links in networks. Nature, 453(7191), 98-101.

Corrado, G. et al. (2016). RNAcommender: Genome-wide recommendation of RNA-protein interactions. Bioinformatics, 32(23), 3627-3634.

Davis, J. and Goadrich, M. (2006). The relationship between precision-recall and roc curves. In Proceedings of the 23rd international conference on Machine learning, pages 233-240.

Gilmer, J. et al. (2017). Neural message passing for quantum chemistry. In 34th International Conference on Machine Learning, ICML 2017, volume 3, pages 2053-2070.

Hentze, M. W. et al. (2018). A brave new world of rna-binding proteins. Nature Reviews Molecular Cell Biology, 19(5), 327.

Kazan, H. et al. (2010). Rnacontext: a new method for learning the sequence and structure binding preferences of rna-binding proteins. PLoS computational biology, 6(7), e1000832.

Kipf, T. N. and Welling, M. (2017). Semi-supervised classification with graph convolutional networks. In 5th Int. Conf. Learn. Represent. ICLR 2017 - Conf. Track Proc., pages 1-14.

Li, Q. et al. (2011). Measuring reproducibility of high-throughput experiments. The annals of applied statistics, 5(3), 1752-1779.

Licatalosi, D. D. et al. (2008). Hits-clip yields genome-wide insights into brain alternative rna processing. Nature, 456(7221), 464-469.

Lichtenwalter, R. N. et al. (2010). New perspectives and methods in link prediction. In Proceedings of the 16th ACM SIGKDD international conference on Knowledge discovery and data mining, pages $243-252$.

Liu, L. et al. (2019). Insight into novel rna-binding activities via large-scale analysis of lncrna-bound proteome and idh1-bound transcriptome. Nucleic acids research, 47(5), 2244-2262.

Maticzka, D. et al. (2014). Graphprot: modeling binding preferences of rna-binding proteins. Genome biology, 15(1), 1-18.

Mikolov, T. et al. (2013a). Distributed representations of words and phrases and their compositionality. In Advances in neural information processing systems, pages 3111-3119.

Mikolov, T. et al. (2013b). Efficient estimation of word representations in vector space. arXiv preprint arXiv:1301.3781.

Muppirala, U. K. et al. (2011). Predicting RNA-Protein Interactions Using Only Sequence Information. BMC Bioinformatics, 12(1), 489.

Muzio, G. et al. (2020). Biological network analysis with deep learning. Briefings in Bioinformatics.

Ozsolak, F. and Milos, P. M. (2011). Rna sequencing: advances, challenges and opportunities. Nature reviews genetics, $\mathbf{1 2}(2), 87-98$.

Pan, X. et al. (2016). Ipminer: hidden ncrna-protein interaction sequential pattern mining with stacked autoencoder for accurate computational prediction. BMC genomics, 17(1), 1-14.

Pan, X. et al. (2019). Recent methodology progress of deep learning for RNA-protein interaction prediction. Wiley Interdiscip. Rev. RNA, 10(6), 1-20. 
Shen, J. et al. (2007). Predicting protein-protein interactions based only on sequences information. Proceedings of the National Academy of Sciences, 104(11), 4337-4341.

Uhl, M. et al. (2017). Computational analysis of clip-seq data. Methods, 118, 60-72.

Uhl, M. et al. (2021). Rnaprot: an efficient and feature-rich rna binding protein binding site predictor. GigaScience, 10(8), giab054.

Van Nostrand, E. L. et al. (2016). Robust transcriptome-wide discovery of rna-binding protein binding sites with enhanced clip (eclip). Nature methods, 13(6), 508-514.

Van Nostrand, E. L. et al. (2020). A large-scale binding and functional map of human RNA-binding proteins. Nature, $\mathbf{5 8 3}(7818), 711-719$.

Wang, L. et al. (2018). Combining high speed elm learning with a deep convolutional neural network feature encoding for predicting protein-rna interactions. IEEE/ACM transactions on computational biology and bioinformatics, 17(3), 972-980.

Wang, L. et al. (2019). Prediction of rna-protein interactions by combining deep convolutional neural network with feature selection ensemble method. Journal of theoretical biology, 461, 230-238.

Wu, Z. et al. (2020). A Comprehensive Survey on Graph Neural Networks. IEEE Transactions on Neural Networks and Learning Systems, $\mathbf{X X}(\mathrm{Xx}), 1-21$.

Yang, Y. et al. (2015). Evaluating link prediction methods. Knowledge and Information Systems, 45(3), 751-782.

Yi, H.-C. et al. (2018). A deep learning framework for robust and accurate prediction of ncrna-protein interactions using evolutionary information. Molecular Therapy-Nucleic Acids, 11, 337-344.

Ying, R. et al. (2018). Graph convolutional neural networks for web-scale recommender systems. In Proceedings of the 24th ACM SIGKDD International Conference on Knowledge Discovery \& Data Mining, pages 974-983.

Ying, R. et al. (2019). Gnnexplainer: Generating explanations for graph neural networks. Advances in neural information processing systems, 32, 9240.

Zhou, J. et al. (2020). Graph neural networks: A review of methods and applications. AI Open, 1, $57-81$. 